\title{
Acupuncture Targeting SIRT1 in the Hypothalamic Arcuate Nucleus Can Improve Obesity in High-Fat-Diet-Induced Rats with Insulin Resistance via an Anorectic Effect
}

\author{
Qing Shu ${ }^{a, c}$ Li Chen ${ }^{b, c}$ Song Wu ${ }^{b, c}$ Jia Lib,c Jianmin Liub,c Ling Xiaoc, e \\ Rui Chen ${ }^{d}$ Fengxia Liang ${ }^{b, c}$ \\ aDepartment of Rehabilitation, Zhongnan Hospital of Wuhan University, Wuhan University, \\ Wuhan, China; ${ }^{b}$ College of Acupuncture, Moxibustion and Orthopedics, Hubei University of \\ Chinese Medicine, Wuhan, China; ${ }^{c}$ Hubei Provincial Collaborative Innovation Center of Preventive \\ Treatment by Acupuncture and Moxibustion, Wuhan, China; ${ }^{\mathrm{d}}$ Department of Traditional \\ Chinese Medicine, Union Hospital, Tongji Medical College, Huazhong University of Science

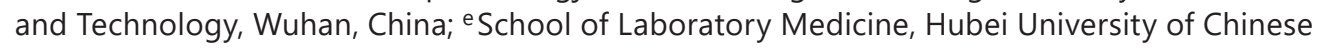 \\ Medicine, Wuhan, China
}

\section{Keywords}

Obesity $\cdot$ Insulin resistance $\cdot$ Electroacupuncture $\cdot$ SIRT1 $\cdot$ FOXO1 $\cdot$ Appetite-related peptide

\begin{abstract}
Objective: To investigate the anorexigenic and anti-obesity effectiveness of electroacupuncture (EA) on high-fat-diet-induced (HFDI) obese rats with insulin resistance (IR) and to reveal the possible mechanisms of EA affecting SIRT1 (silent mating type information regulation 2 homolog 1) in the central nervous system (CNS). Methods: We divided 60 rats into 6 groups. All interventions, including EA and intracerebroventricular administration, were performed after 8 weeks of model establishment. We tested obesity phenotypes like body weight (BW) gain; food intake; and IR levels including glucose infusion rate, intraperitoneal insulin tolerance test (IPITT), and intraperitoneal glucose tolerance test (IPGTT) during treatment. We detected protein expression and microscopic locations in hypothalamic SIRT1, the transcription factor FOXO1 (forkhead box protein O1), acetylated FOXO1 (Ac-FOXO1), pro-opiomelanocortin (POMC), and neuropeptide Y (NPY) via Western blotting and immunofluorescence, and monitored gene expression by real-time polymerase chain reaction. Results: Like the SIRT1 agonist, EA suppressed BW gain and IR levels in obese rats, but this was only partially blocked by the SIRT1 antagonist. EA could upregulate protein expression of hypothalamic SIRT1 and downregulate the acetylation level of FOXO1 in the hypothalamic arcuate nucleus (ARC),
\end{abstract}

Fengxia Liang

Hubei University of Chinese Medicine

1 Tanhualin, Wuchang District,

Wuhan, Hubei 430061 (China)

E-Mail fxliang5@hotmail.com
Rui Chen

Department of Traditional Chinese Medicine, Union Hospital

Tongji Medical College, Huazhong University of Science and Technology

1277 Jiefang Street, Wuhan, Hubei 430022 (China)

E-Mail unioncr@163.com 
which decreased gene expression of NPY and increased that of POMC. The agonist targeted the hypothalamic SIRT1 gene, unlike EA, which targeted posttranscriptional regulation. Conclusion: EA could improve obesity in HFDI rats with IR via its anorectic effect. This effect targeted posttranscriptional regulation of the SIRT1 gene, which induced upregulation of ARC FOXO1 deacetylation and mediated the gene expression of POMC and NPY.

(C) 2020 The Author(s)

Published by S. Karger AG, Basel

\section{Introduction}

Overweight and obesity are defined as abnormal or excessive fat accumulation that may impair health, which is measured by body mass index (BMI) [1]. According to GBD (Global Burden of Disease) 2015 Obesity Collaborator data published by the World Health Organization (WHO), in 2015 more than 1.9 billion adults (defined as 18 years and older) were overweight (BMI $\geq 25$ ); of these, 603.7 million were obese (BMI $\geq 30$ ), as were 107.7 million children [2]. If these trends continue, by 2025 global obesity prevalence will reach $18 \%$ in men and surpass $21 \%$ in women, while severe obesity will surpass $6 \%$ in men and $9 \%$ in women [3]. Obesity is one of the most serious risk factors in the development of type 2 diabetes mellitus, cardiovascular diseases, and even some types of cancer and other adverse pathological conditions [4]. Insulin resistance (IR) is the key pathological process linking obesity and type 2 diabetes mellitus, modulated by abnormal adipose-tissue deposits that induce secretion of nonesterified fatty acids, glycerol, hormones such as leptin and adiponectin, and proinflammatory cytokines [5]. A 7\% reduction in body weight (BW) can lower the risk of diabetes by $58 \%$ [6].

Existing anti-obesity pharmacology has been limited by side effects, including depression, suicidal thoughts [7], cardiovascular complications, and stroke [8, 9]. In addition to drugs and bariatric surgery, caloric restriction [10] and physical activity [11] have proven effective in treating obesity and related IR. Controversially, exercise without caloric restriction does not produce weight loss [12], which indicates that restriction of food intake is the basis of weight management.

Acupuncture, a traditional eastern medical therapy with a long history, is one of the most popular complementary and alternative therapies in western countries, with increasingly potent evidence that it can treat obesity [13]. A systematic review indicates that acupuncture is effective in the treatment of obesity [14], as well as its related IR [15]. In addition, nutritional satisfaction and anorexia are the most pronounced proprioceptions after treatment with acupuncture [16], but the inherent mechanisms of these phenomena have not been revealed and therefore need more investigation.

The hypothalamus is the center of food intake modulation and energy metabolism [17]. The arcuate nucleus (ARC) is located in the base of the hypothalamus and adjoins the third ventricle, which can sense changes in nutrition via the peripheral circulation. Pro-opiomelanocortin (POMC) and neuropeptide Y (NPY) neurons, the primary sensors and mediators in the ARC, have opposite functions in the regulation of appetite [18]. Sirtuin1 or SIRT1 (silent mating type information regulation 2 homolog 1), a deacetylase which is involved in lifespan extension induced by caloric restriction [19], has recently been found to play a prominent role in obesity and related IR [20]. The expression of hypothalamic SIRT1 was increased on feeding, and this induction is abrogated in diet-induced obese mice [21]. Overexpression of SIRT1 in POMC neurons stimulated energy expenditure, whereas overexpression in agouti-related protein (AgRP) neurons suppressed food intake [22]. FOX01 (forkhead box protein 01), one of the deacetylated substrates of SIRT1 [23], is expressed in metabolic tissue such as hepatic, skeletomuscular, adipose, and hypothalamic tissue [24, 25], which can regulate AgRP expression in ARC [21]. 
EA has been proven effective in restricting caloric intake and improving obesity via POMC and NPY regulation [26,27]. The underlying mechanism of acupuncture has yet to be clearly established, especially its effects on the central regulation of food intake. Our previous study showed that EA can improve insulin sensitivity in obese diabetic mice by activating SIRT1 in skeletal muscle [28]. Based on this, we designed the present study to figure out the EA-induced specific regulation of the SIRT1/FOX01 signaling pathway and related peptides in the hypothalamic ARC.

\section{Materials and Methods}

\section{Animal Models and Allocation}

All animal procedures were performed according to the Declaration of Helsinki (European Union guidelines on the use of animals in scientific experiments) and Animal Research: Reporting of In Vivo Experiments (ARRIVE) guidelines [29].

We studied 90 male Wistar rats (8 weeks old and weighing 205-255 g) from the Hubei Research Center of Laboratory Animals (No. 42000600013006). All rats were placed in an environment controlled for temperature $\left(22 \pm 2{ }^{\circ} \mathrm{C}\right)$ and humidity $(50 \pm 10 \%)$ with a 12 -h light/12-h dark cycle in the Experimental Animal Center of Hubei University of Chinese Medicine, and food and water was provided ad libitum for the duration of the study. After a 1-week adaptation to the facility, we randomly selected 15 rats and fed them a standard rodent $\operatorname{diet}$ ( $3.8 \mathrm{kcal} / \mathrm{g} \mathrm{BW} ; 10 \%$ fat, $70 \%$ carbohydrates, and 20\% protein). We fed the other 75 rats a high-fat diet (5.5 kcal/g BW; 46.5\% fat, 38.5\% carbohydrates, and 15\% protein) [30].

We weighed all rats after feeding them for 8 weeks. The standard for inclusion in our obese rat model was a $20 \%$ increase in weight compared to rats fed a standard rodent diet. We used a hyperinsulinemic-euglycemic clamp to assess insulin sensitivity in both groups after 8 weeks. We included diet-induced obesity-prone (DIO-P) rats which met the inclusion standard of being $20 \%$ heavier than rats fed the standard rodent diet in our study while excluding dietinduced obesity-resistant (DIO-R) rats. Eventually, for our normal group, we randomly selected 10 rats from the 15 that had been fed a standard rodent diet (normal group, NG; $n=10$ ). Then we randomly selected 50 of the 61 DIO-P rats and divided them into a model group (MG; $n=$ 10), an EA group (EA; $n=10)$, a sham operation group (SO; $n=10)$, an agonist group (AG; $n=$ 10 ), and an EA-plus-antagonist group (EA $+\mathrm{AN} ; n=10$ ). We randomly selected 3 obese rats in each obese rat group for the hyperinsulinemic-euglycemic clamp test in the process of allocation. The results showed that all 3 obese rats randomly selected had IR.

\section{Animal Interventions}

EA Methods

Before EA treatment, we fixed rats using an instrument made specifically for this experiment to keep them calm. We applied EA at the acupoints of Zusanli (ST36), Guanyuan (CV4), Zhongwan (CV12), and Fenglong (ST40) using $0.30 \times 25 \mathrm{~mm}$ needles (Global, China). We based the acupoint locations on our measurements of body length according to existing standards [31] as described previously [28, 32, 33] (Fig. 1A; online suppl. 2; for all online suppl. material, see www.karger.com/doi/10.1159/000503752).

We linked CV4 and CV12 with 2 electrodes of a Han's acupoint nerve stimulator (HANS LH202H, China), while we linked ST36 and ST40 with 2 other electrodes. We switched the acupoints to a contralateral leg every other day. We electrically stimulated the acupoints with successive waves of $2 \mathrm{~Hz}$, adjusting intensity to produce local muscle contractions that varied from 0.5 to $1 \mathrm{~mA}$ [28]. Rats in the EA and EA + AN groups received EA treatment for $10 \mathrm{~min}$ every other day. The other groups, which did not receive EA, were fixed at the same time. 
Intracerebroventricular Administration in the Third Ventricle

We fixed rats in the SO, AG, and EA + AN groups in a stereotaxic apparatus (RWD Life Science and Technology, China) and anesthetized them with isoflurane. In accordance with the 6th edition of The Rat Brain in Stereotaxic Coordinates [34], we chose a point within the dorsal third ventricle (D3V, AP: $-1.56 \mathrm{~mm}$, ML: $0 \mathrm{~mm}$, DV: $3.8 \mathrm{~mm}$ ). We obtained 5-mm paraffin sections and performed Nissl staining to confirm the precise locations of the cannulae (Fig. 1B).

In order to investigate whether ventricular cannulation will affect the role of EA in improving obesity, one group that received intracerebroventricular administration of artificial cerebrospinal fluid (ASCF) plus EA treatment (EA + ASCF) was compared to the EA + AN group. The results showed that rats in the EA + ASCF group did not differ in Lee's index and food intake from the EA group, while both groups (EA + ASCF group and EA group) were significantly different from the EA + AN group (Fig. 1C). These results suggested that cannulation did not affect the EA in improving obesity and food intake.

Rats in the SO, AG, and EA + AN groups were fed separately and given 1 week after the operation to adapt to the cannulae. At the same time of treatment in the EA group, we intracerebroventricularly injected rats in the SO group with ASCF, rats in the AG group with the SIRT1 agonist SRT1720 (1 $\mu \mathrm{g} / \mu \mathrm{L}, 2 \mu \mathrm{L}$; Selleck, US) [35], and rats in the EA + AN group with the SIRT1 antagonist EX-527 (1 $\mu \mathrm{g} / \mu \mathrm{L}, 5 \mu \mathrm{L}$; Selleck) before EA treatment (Fig. 1D) [36].

\section{Parameter Detection}

Body Mass, Lee Index, Food Intake, Fasting, and Postprandial Blood Glucose and

Serum Insulin

We obtained body mass, nasoanal length, and food intake measurements $0,2,4,6$, and 8 weeks after commencement of EA treatment. Mathematically, we expressed the Lee index as follows [37]:

$$
\frac{\sqrt[3]{\text { body weight }(\mathrm{g}) \times 1,000}}{\text { nasoanal length }(\mathrm{cm})} .
$$

Fasting blood glucose (FBG) levels were measured by the tail snipping method at 8:00 a.m. after $8 \mathrm{~h}$ of fasting and postprandial blood glucose (PBG) at 8:00 a.m. after rats had access to food ad libitum for a whole night using a glucose testing machine (OneTouch, US). After the 8 -week intervention, we anesthetized the rats with $10 \%$ chloral hydrate, collected serum from their hearts, and determined insulin levels using a commercial ELISA kit (H203; Nanjing Jiancheng Bioengineering Institute, China).

\section{Insulin Sensitivity}

We performed an intraperitoneal insulin tolerance test (IPITT) and intraperitoneal glucose tolerance test (IPGTT) on all rats after 6 weeks of intervention. For the IPITT, after $8 \mathrm{~h}$ of fasting, we injected rats intraperitoneally with 1 unit/kg insulin solution; we collected blood samples after tail snipping to determine insulin levels prior to and 30, 60, 90, and $120 \mathrm{~min}$ after insulin administration. For the IPGTT, after $12 \mathrm{~h}$ of fasting, all rats received an intraperitoneal injection of $50 \%$ glucose $(2 \mathrm{~g} / \mathrm{kg}$ ), and blood samples were again collected to determine glucose levels before and 30, 60, 90, and $120 \mathrm{~min}$ after insulin administration. Then, we calculated the area under the curve (AUC) of each group using the approximate trapezoidal method [38]:

$$
\mathrm{AUC}=\left(\frac{F B G+P B G_{120}}{2}+P B G_{30}+P B G_{60}+P B G_{90}\right) \times 30 \mathrm{~min} .
$$




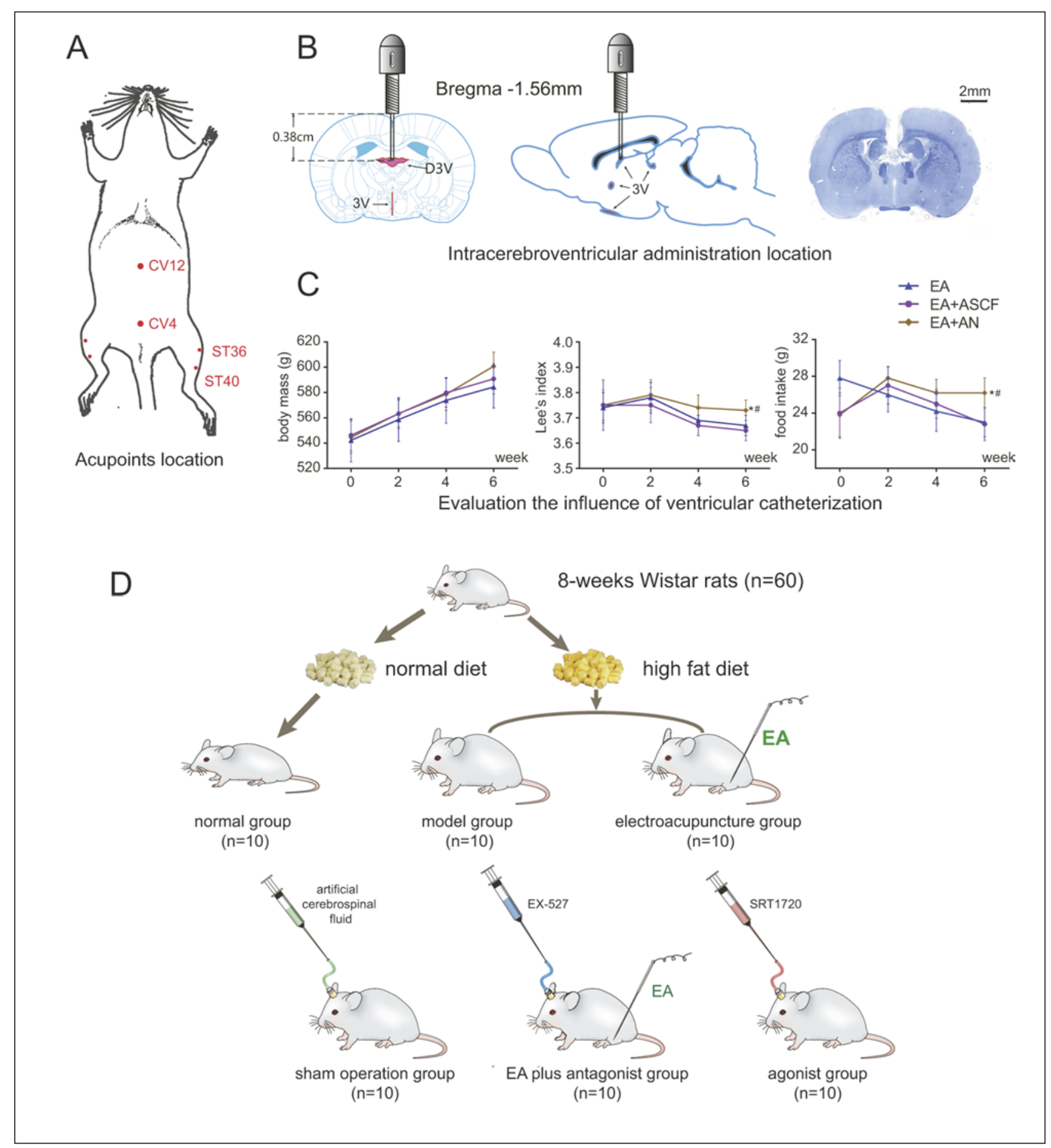

Fig. 1. A Acupoint locations. B Intracerebroventricular administration. C Evaluation of the influence of ventricular catheterization. D Animal allocation $\left({ }^{*} p<0.05\right.$ vs. EA, ${ }^{*} p<0.05$ vs. EA + ASCF).

To assess IR levels, we randomly selected 3 rats from each group and performed caudalartery and -vein hyperinsulinemic-euglycemic clamp on them before and after treatment for 7 weeks and calculated mean glucose infusion rate (GIR) based on GIR from 60-120 min (online suppl. 2). 
Table 1. Real-time PCR primer sequences

\begin{tabular}{|c|c|c|}
\hline Gene & Forward & Reverse \\
\hline SIRT1 & 5'-TGCCATCATGAAGCCAGAGA-3' & 5'-CATCGCAGTCTCCAAGAAGC-3' \\
\hline F0X01 & 5'-CAGCAAATCAAGTTATGGAGGAT-3' & 5'-CTGGCATGACTGAGTTAGGG-3' \\
\hline POMC & 5'-ATAGACGTGTGGAGCTGGTG-3' & 5'-GGGGATTTTCAGTCAAGGGC-3' \\
\hline NPY & 5'-AACAAACGAATGGGGCTGTG-3' & 5'-GTGTCTCAGGGCTGGATCTC-3' \\
\hline$\beta$-Actin & 5'-CACGATGGAGGGGCCGGACTCATC-3' & 5'-TAAAGACCTCTATGCCAACACAGT-3' \\
\hline
\end{tabular}

\section{Western Blotting}

All rats were anesthetized and sacrificed by cervical dislocation after 8 weeks of treatment. We froze the hypothalami in liquid nitrogen after stripping them from the rat brains and stored them at $-80^{\circ} \mathrm{C}$. Hypothalamus tissue was detected applying a standard Western blotting (WB) protocol. The primary antibody concentrations were as follows: SIRT1 (1:8,000, ab110304; Abcam, UK), FOXO1 (1:2,000, ab52857; Abcam), Ac-FOXO1 (1:200, D-19; Santa Cruz Technology, USA), POMC (1:200, FL-267; Santa Cruz Technology), and NPY (1:1,000, D7Y5A; Cell Signaling Technology, USA). Images were obtained using a ChemiDoc XRS+ System (Bio-Rad Laboratories, USA). We used the housekeeping protein $\beta$-actin (1:5,000; Proteintech, Manchester, UK) for normalization. We performed WB in triplicate for all target proteins. Protein expression was calculated based on the target protein and $\beta$-actin ratios of optical density (OD), which we analyzed using Gel-Pro Analyzer software, version 4.0 (Polaroid Corporation, USA).

\section{Double-Labeled Immunofluorescence Staining}

We performed double-labeled immunofluorescence (IF) staining of SIRT1/FOXO1, SIRT1/Ac-F0X01, FOX01/POMC, and Ac-FOX01/NPY to investigate protein interaction in the hypothalamic ARC. Rat brain paraffin sections in the coronal plane were processed with standard antigen retrieval and incubated with primary antibody SIRT1 $11: 2,000$, mouse, ab110304; Abcam), FOX01 (1:200, rabbit, ab39670; Abcam), and Ac-FOXO1 (1:200, rabbit, D-19; Santa Cruz Technology). Then, we exposed the sections to secondary antibodies (SIRT1 and FOX01 were labeled with cyanine 3 [CY3] at 1:50, Ac-FOXO1 with fluorescein isothiocyanate [FITC] for 1:50). After blocking in goat or donkey serum a second time, sections were incubated with the secondary primary antibody, including FOX01, Ac-FOXO1, POMC (all 1:200, chicken, ab14064; Abcam), and NPY (1:500, sheep, ab6173; Abcam). We then exposed the sections to secondary antibodies (FOXO1 and FOXO1 were labeled with FITC at 1:50, POMC with Fluor-488 at 1:1,000, and NPY with Fluor-555 at 1:1,000). We stained nuclei with 4',6-diamidino-2-phenylindole (DAPI) and acquired images using an Olympus BX53 digital fluorescence microscope (Olympus Corporation, Japan). Specificity of all antibodies was verified using a negative control. We replaced the primary antibodies with PBS or serum from the same source as the primary antibody. In addition, all the antibodies were validated in published studies.

\section{Real-Time Polymerase Chain Reaction}

We isolated total RNA of rat hypothalami stored at $-80^{\circ} \mathrm{C}$ with an AZfresh total RNA extraction kit (Azanno Biotech, Gothenburg, Sweden) and determined RNA concentrations at an absorbance ratio of $260 / 280 \mathrm{~nm}$. We then reverse transcribed an aliquot ( $1 \mu \mathrm{g}$ ) of extracted RNA into first-strain complementary DNA (cDNA) using a ReverTra Ace qPCR RT kit (FSQ101; Toyobo). We quantified gene expression of SIRT1, FOX01, Ac-FOX01, POMC, and NPY by using a SYBR Green real-time PCR Master Mix Plus (QPK-212; Toyobo) and standard protocol. 
Shu et al.: Acupuncture Targeting SIRT1 and Obesity/Insulin Resistance

Measurements were conducted in triplicate under standard reaction conditions, and normalization was ensured to $\beta$-actin. We obtained primaries from Tsing Ke Biology Technology Company (Wuhan, China). All temperature circulation and gene amplification were processed in a CFX96 Touch real-time PCR detection system (Bio-Rad). All RT-PCR assays and primer sequences are shown in Table 1.

\section{Statistical Analysis}

We repeated WB of the hypothalami in each group 3 times and included the OD of each target protein in our statistical analysis. For IF, we randomly selected 5 sections from each group for analysis. We calculated the positive cells in the ARC at $\times 400$ magnification and determined relative protein expression by the ratio of positive cells (OD value of the fluorescence exceeds $50 \%$ of the background) to total cells (count by calculating DAPI-stained nuclei). The difference in gene expression was demonstrated by the relative gene expression compared to the normal group, which we calculated using CFX Manager Software, version 3.0 (Bio-Rad Laboratories).

Analysis was performed by blinded biostatisticians using SPSS software version 20.0 (IBM SPSS Statistics, USA). We used repeated-measure analysis of variance (ANOVA) to compare body mass, Lee index, food intake, FBG, and PBG at different time points $(0,2,4,6$, and 8 weeks) within each group to show improvements in obesity and energy metabolism effected by treatments. Post hoc multiple comparisons with the Turkey method were conducted if there were differences in any group. In addition, we used one-way ANOVA to compare the differences in body mass, Lee index, food intake, FBG, PBG, and AUC of IPGTT/ IPITT between groups at different time points. We then analyzed changes in these measurements plus serum insulin, GIR, OD of protein bands, percentage of positive cells in IF, and relative gene expression among the 6 groups by one-way ANOVA. To assess differences between 2 groups, we conducted post hoc multiple comparisons with the Turkey method if there were differences among all 6 groups. Values of $p<0.05$ were considered statistically significant, and tests were 2 -sided.

\section{Results}

EA Improved Obesity and Increased Insulin Sensitivity but the SIRT1 Antagonist Could Partially Suppress this Effect

EA Suppressed Body Mass Gain and Food Intake but the SIRT1 Antagonist Partially Inhibited These Effects

Before and after 8 weeks of treatment, body mass in the NG group was significantly lower than in the other 5 groups. Body mass in the MG and SO groups showed the most significant increase, while it slowed in rats that received EA $(p=0.0028)$. Rats in the EA + AN group, which received both EA and SIRT1 antagonist, did not gain significantly more weight than those in the EA group ( $p=0.66$ ), while body mass in the AG group did not show significant growth during the 8 weeks of treatment (Fig. 2A). Before treatment, the Lee index was significantly lower in the NG group than in the other groups that were fed a high-fat diet. After the course of the 8-week treatment, the Lee index was increased in the MG and SO groups versus before treatment $(p<$ 0.05 ), while it was not different in the EA and AG groups. The Lee index was lower in the EA and AG groups than in the MG and SO groups, while the SIRT1 antagonist suppressed the EA effect on the Lee index ( $p=0.011$ ) (Fig. 2B). Food intake in the high-fat diet-induced (HFDI) obese rats was significantly higher than in the NG rats. During the course of the 8-week treatment, there was no significant change in the food intake of the NG and MG groups. The food intake of EA rats was lower than that of MG rats after 4 weeks of treatment ( $p=0.0099)$, while the SIRT1 antag- 
A

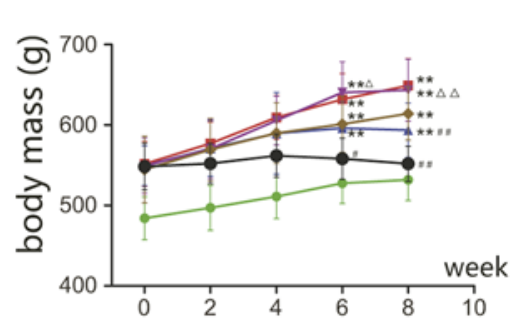

B

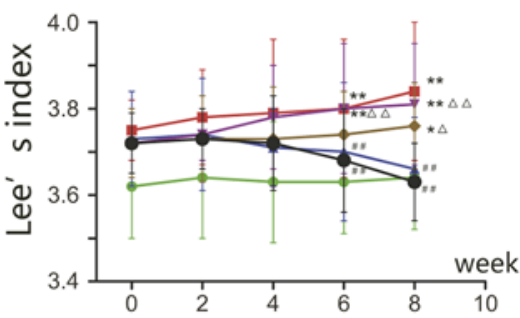

C

D
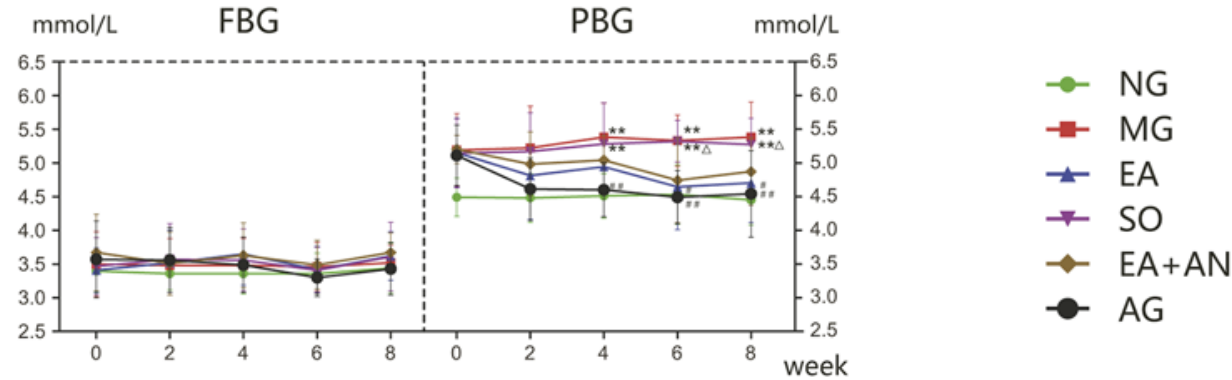

E

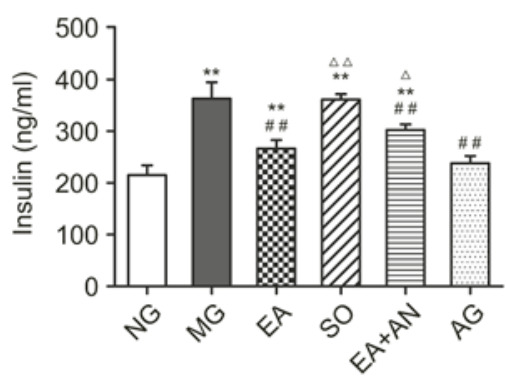

$\mathrm{F}$
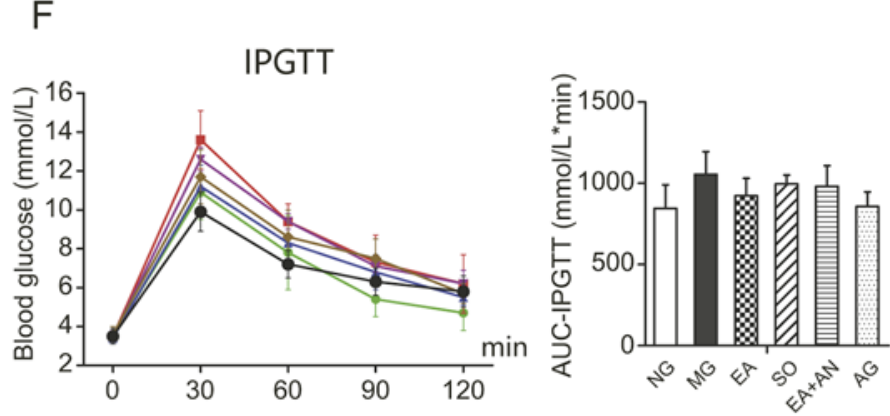

$\mathrm{H}$

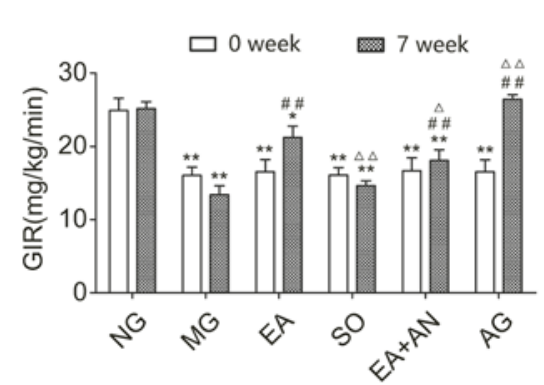

G
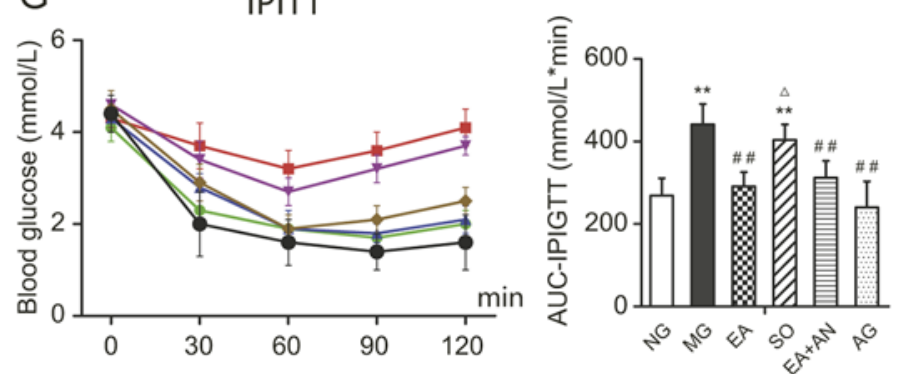

Fig. 2. Alterations in body mass (A), Lee index (B), food intake (C), FBG/PGB (D), insulin sensitivity (E), IPGTT (F), GIR (H), and IPITT (G). ${ }^{*} p<0.05,{ }^{* *} p<0.01$, vs. NG; ${ }^{\#} p<0.05,{ }^{\# \#} p<0.01$, vs. MG; ${ }^{\Delta} p<0.05$, $\Delta \Delta p<0.01$, vs. EA. NG, normal group; MG, model group; EA, electroacupuncture group; SO, sham operation group; EA + AN, EA-plus-antagonist group; AG, agonist group; AUC, area under the curve. 
onist partially inhibited these effects after 8 weeks of treatment ( $p=0.0404)$. However, the rats in the EA, AG, and EA + AN groups, which were cannulated, showed a significant decrease in food intake after surgery ( 0 weeks) but recovered after 2 weeks. At the end of treatment, the AG group had the lowest food intake, which was even lower than that of the NG group, while there were no significant differences between the NG and EA groups (Fig. 2C).

EA Could Improve PBG and Serum Insulin Levels with No Effect on FBG, but the SIRT1 Antagonist Could Still Partially Suppress This Effect

After they were fed a high-fat diet, the PBG of rats rose to a certain degree but remained within the normal range. During the course of the 8-week treatment, PBG did not show a statistically significant difference in any group. After 6 weeks of treatment, PBG was lower in the EA and AG groups than in the MG group until the end of treatment at 8 weeks $(p<0.05)$. There was no significant difference in the degree of PBG decline among the EA, AG, and EA + AN groups after 8 weeks of treatment (Fig. 2D). Compared with the NG group, the MG and SO groups showed a phenotype of hyperinsulinemia, which EA and the SIRT1 agonist were able to reverse to some extent. Consistent with the effects on BW and food intake, the SIRT1 antagonist still attenuated the antihyperinsulinemic effect of EA (Fig. 2E).

EA Can Improve Insulin Sensitivity in HFDI Obese Rats - An Effect That Was Also Attenuated by the SIRT1 Antagonist

We performed IPGTT and IPITT to investigate the effectiveness of EA in improving IR and the possible mechanism thereof. EA and the SIRT1 agonist resulted in lower blood glucose levels in the NG group 30 min after IPGTT, which were statistically different from those of the MG group $(p<0.05)$. Blood glucose levels were not significantly different 60 min after injection $(p>0.05)$ in the study groups (Fig. 2F). The AUC of IPGTT did not show significant differences in any of the 6 groups $(p<0.05)$. As depicted in Figure 2G of IPITT, blood glucose levels showed a transient and smaller decline in the MG and $S 0$ groups than those in the EA, AG, and $\mathrm{EA}+\mathrm{AN}$ groups, which increased 60 min after intraperitoneal injection. During the course of treatment, rats in the EA, AG, and EA + AN groups showed a sharper and greater decline in blood glucose levels than did those in the MG and SO groups. The IPITT AUC of the EA, EA + AN, and AG groups was significantly lower than that of the MG and SO groups, in which the SIRT1 antagonist did not significantly inhibit the effect of EA (Fig. 2G). We used a hyperinsulinemic-euglycemic clamp to evaluate insulin sensitivity precisely and reliably. Before treatment, GIR in the NG group was significantly higher than that in HFDI obese rats, which indicated that the latter demonstrated IR. After 7 weeks of treatment, EA and the SIRT1 agonist significantly increased the GIR of obese rats with IR, while the SIRT1 antagonist was able to partially attenuate the effect of EA (Fig. $2 \mathrm{H}$ ).

EA Upregulated Protein Expression of SIRT1, Downregulated Acetylation of FOXO1, and Mediated the Downstream Appetite-Related Neuropeptide, an Effect That the SIRT1 Antagonist also Suppressed

After feeding the MG and SO rats a high-fat diet, protein expression of hypothalamic SIRT1 was suppressed in these rats and upregulated after treatment with EA or intracerebroventricular administration of SRT1720. Rats that received EA plus EX-527 injections showed a lower expression of hypothalamic SIRT1 than rats that received EA alone. Protein expression of hypothalamic FOX01 showed the same tendency with SIRT1, which was highly expressed in the EA and AG groups and less so in the MG and SO groups. Administration of EX-527 could suppress the effect of EA on hypothalamic FOX01 activation. In contrast, hypothalamic Ac-FOXO1 showed low expression in rats fed a normal diet and was highly expressed in HFDI obese rats, indicating high deacetylation activity in the NG group. Ac-FOXO1 was downregulated after administration 


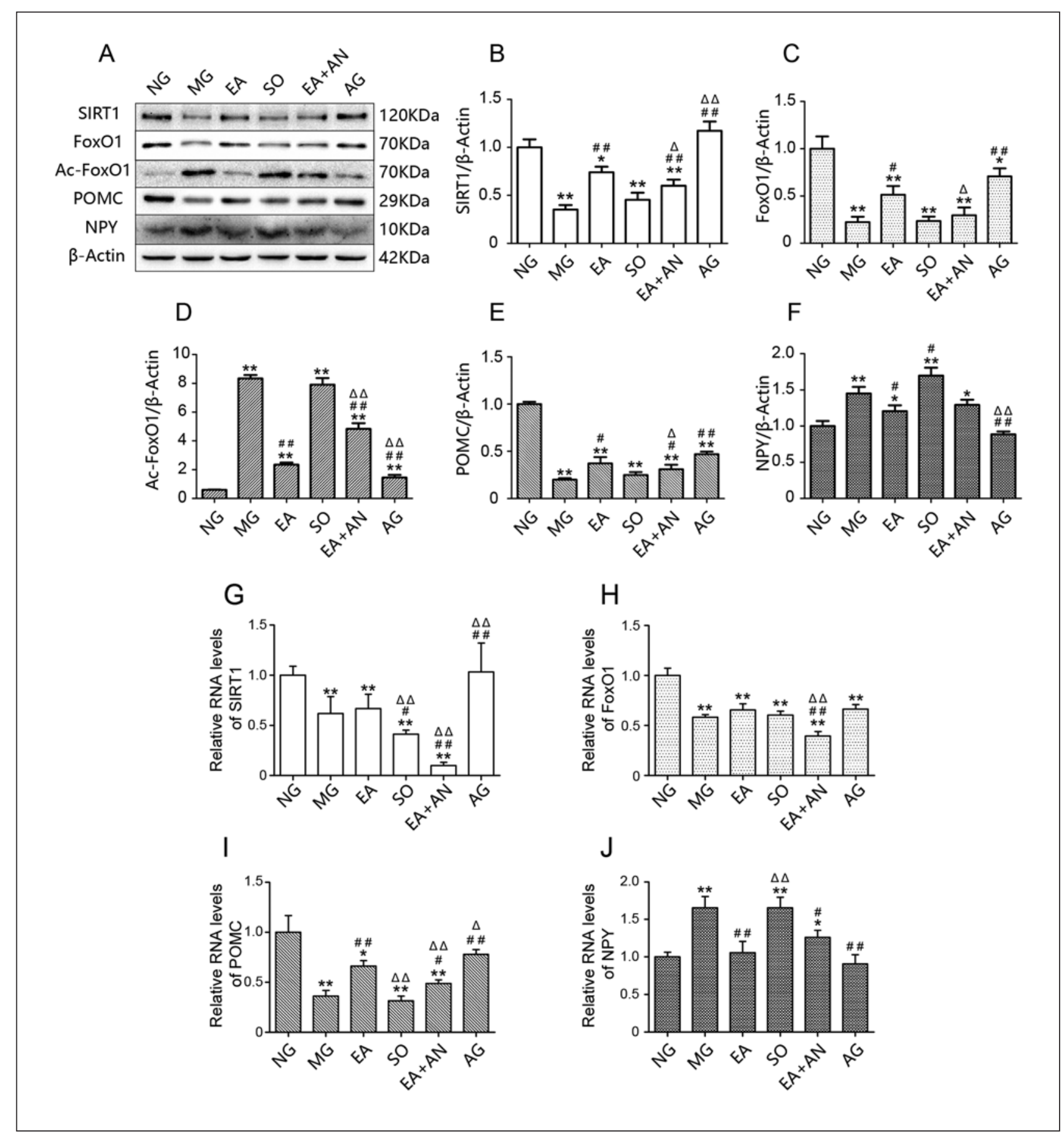

Fig. 3. A-J Protein expression and relative mRNA levels of SIRT1, FOX01, Ac-FOXO1, POMC, and NPY in the hypothalamus. ${ }^{*} p<0.05,{ }^{* *} p<0.01$, vs. NG; ${ }^{\#} p<0.05$, \#\# $p<0.01$, vs. MG; ${ }^{\Delta} p<0.05,{ }^{\Delta \Delta} p<0.01$, vs. EA. SIRT1, silent mating type information regulation 2 homolog 1; FOX01, forkhead box protein 01; Ac-FOX01, acetylated FOX01; NPY, neuropeptide Y.

of EA and the SIRT1 agonist, indicating upregulation of suppressed deacetylation activity. The effectiveness of EA was consistently attenuated when EA was combined with EX-527. In each group, hypothalamic POMC expression was consistent with SIRT1 and FOXO1 expression, while hypothalamic NPY expression was similar to Ac-FOXO1 expression (Fig. 3A-F). 
Shu et al.: Acupuncture Targeting SIRT1 and Obesity/Insulin Resistance

Modulation of EA Activates the Deacetylation of SIRT1 Located at Hypothalamic ARC

As shown in Figure 4A and online supplementary Figure 1, SIRT1 and FOXO1 were both expressed throughout the entire hypothalamus and more aggregated in the ARC. SIRT1 and FOX01 showed far more expression in the hypothalamic ARC of rats fed a normal diet than in those fed a high-fat diet. After treatment with EA or SRT1720, both SIRT1 and FOX01 were upregulated in the hypothalamic ARC. However, EX-527 partially attenuated the EA-mediated upgrading effect of SIRT1 and FOX01. High-fat-diet-mediated SIRT1 inhibition resulted in upregulation of AC-FOXO1 expression in the ARC. After 8 weeks of treatment, the low level of deacetylation in the ARC was activated by EA or SRT1720, while EX-527 attenuated the deacetylation effect of EA in the EA + AN group. As summarized in Figure 4B and online supplementary Figure 2, deacetylation activation was most pronounced in the NG and AG groups, secondhighest in the EA group, lower in the EA + AN group, and lowest in the MG and SO groups.

As shown in Figure 4C and online supplementary Figure 3, upgrading of FOXO1 in the hypothalamic ARC mediated the high expression of POMC, which has the function of suppressing food intake. In contrast, HFDI low expression of FOXO1 was associated with downregulation of POMC in the hypothalamic ARC. EA- and SRT1720-mediated FOXO1 activation induced upregulation of POMC in the ARC, while EX-527 suppressed the EA-induced effect. In contrast, HFDI hyperacetylation of FOXO1 mediated upregulation of NPY, which is expressed throughout the hypothalamus. The deacetylation of the EA- and SRT1720-induced low level of Ac-FOXO1 mediated NPY downregulation in the hypothalamus, an effect of EA also attenuated by EX-527 (Fig. 4D; online suppl. Fig. 4).

\section{EA Mediated Gene Expression of POMC and NPY but Not That of SIRT1 or FOXO1}

The goal of investigating the gene expression of SIRT1 and mediated downstream molecules was to determine the subcellular localization of EA. As shown in Figure 3G, gene expression of SIRT1 in rats fed a normal diet was significantly higher than that in rats fed a high-fat diet. However, SRT1720 was found to upregulate gene expression of SIRT1 in obese and IR rats, but EA did not, which was consistent with the MG group. Furthermore, EA plus EX-527 significantly downregulated gene expression of SIRT1, indicating that this suppression effect was produced by the SIRT1 antagonist. As shown in Figure 3H, I, the hypothalami of rats fed a normal diet showed high expression of FOXO1 and low expression of Ac-FOXO1, while those of rats fed a high-fat diet showed the opposite. Interestingly, neither EA nor SRT1720 could upregulate the low deacetylation level of FOX01 gene expression. Rats fed a normal diet showed high gene expression of POMC and low gene expression of NPY, while those fed a high-fat diet showed the opposite phenotype. Rats treated with EA or SRT1720 showed upregulation of POMC gene expression and downregulation of NPY gene expression. EX-527 could also suppress the effect of EA, which was consistent with protein expression (Fig. 3J). All gene expression results suggested that EA and SRT1720 activated SIRT1 and related deacetylation via a different mechanism. SRT1720 targeted regulation of SIRT1 gene expression, while EA targeted activation of posttranscriptional SIRT1.

\section{Discussion}

HFDI obesity is always accompanied by an increase in insulin secretion and induction of IR [5]. Adipocytes and suppressed insulin receptor substrate tyrosine phosphorylation in the liver and skeletal muscle both produce nonesterified fatty acids and induce IR [39]. In this study, HFDI obese rats showed a marked increase in IR as determined by the clamp method.

POMC and NPY are appetite-related neuropeptides located in the hypothalamic ARC with opposite functions. POMC-positive neurons secrete $\alpha$-melanocyte-stimulating hormone and 


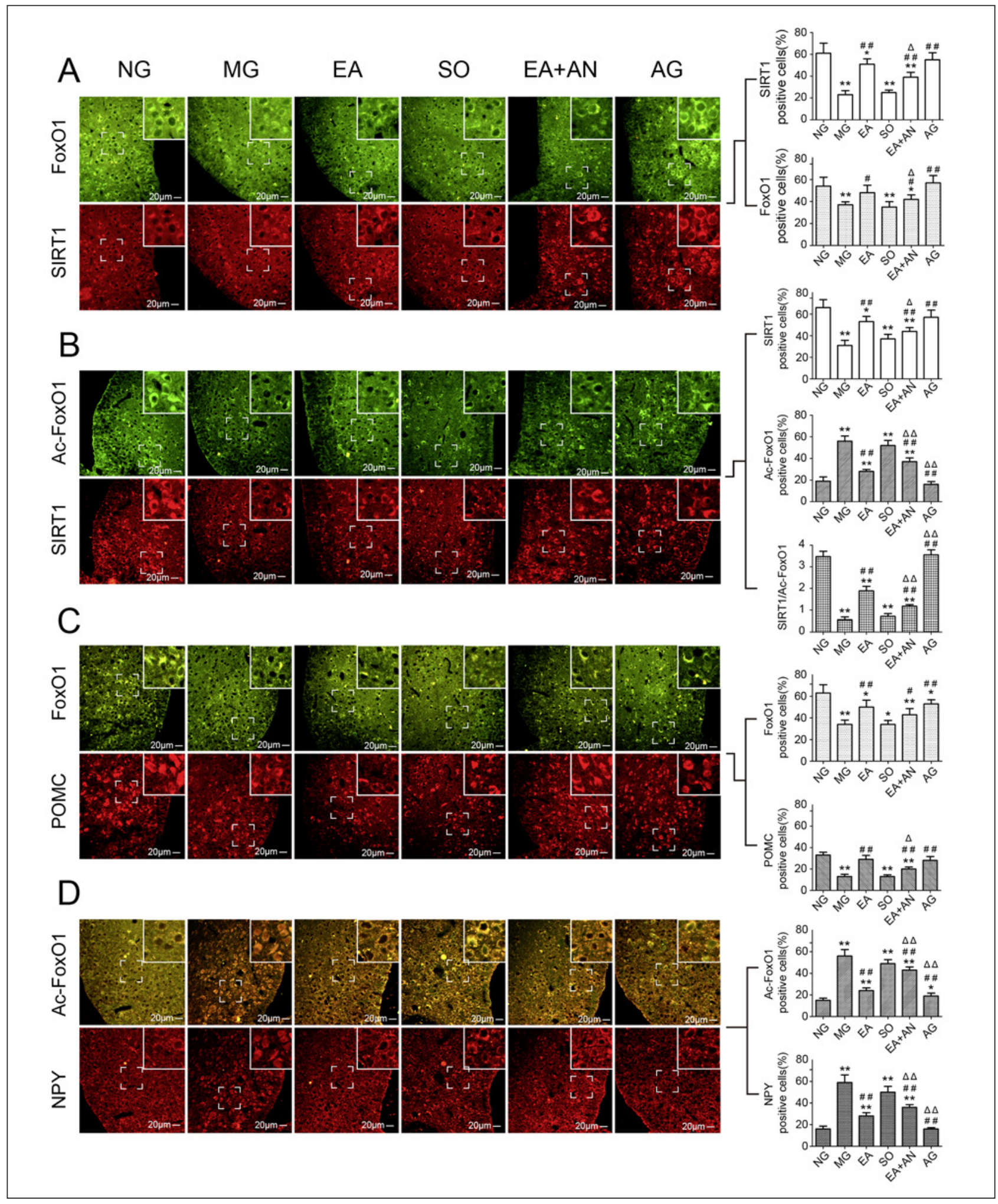

Fig. 4. Double-labeled immunofluorescence of SIRT1/FOX01 (A), SIRT1/Ac-FOX01 (B), FOX01/POMC (C), and Ac-FOX01/NPY (D) in hypothalamic ARC $\left({ }^{*} p<0.05,{ }^{* *} p<0.01\right.$, vs. NG; ${ }^{\#} p<0.05,{ }^{\# \#} p<0.01$, vs. MG; ${ }^{\Delta} p<0.05,{ }^{\Delta \Delta} p<0.01$, vs. EA). SIRT1, silent mating type information regulation 2 homolog 1; FOXO1, forkhead box protein 01; Ac-FOX01, acetylated FOX01; NPY, neuropeptide Y; ARC, arcuate nucleus. 
Shu et al.: Acupuncture Targeting SIRT1 and Obesity/Insulin Resistance

restrain appetite by binding to melanocortin $3 / 4$ receptor in the paraventricular nucleus [40]. NPY, which is secreted from NPY-positive neurons, performs an orexigenic function by binding to NPY Y1 and Y5 receptors in the lateral hypothalamic area [41]. Leptin in the peripheral cycle can directly penetrate the third ventricle and bind to the leptin receptor located on the POMC/cocaine- and amphetamine-regulated transcript and NPY/AgRP neuronal-cell membranes in the hypothalamic ARC, which regulate food intake and energy homeostasis [42]. In obesity and IR, there is an incontrollable negative feedback loop in the hypothalamus, which causes hyperleptinemia, hyperinsulinemia, and appetite hyperactivity [43].

FOX01, which is highly expressed in the hypothalamus, can negatively regulate the transcription of POMC via direct binding to its promoter or inhibitor signal transducer and activator of transcription 3. In addition, FOX01 overexpression stimulates transcription of orexigenic NPY/AgRP through the phosphatidylinositol 3-kinase/protein kinase B (Akt) signaling pathway [25]. Furthermore, the deacetylation level of FOXO1 in the hypothalamus was shown to determine the transcription of downstream orexin-related genes; acetylated FOXO1 promoted the transcription of NPY and AgRP [44], while deacetylated FOXO1 upregulated gene expression of POMC [45].

SIRT1, which is expressed in several insulin-sensitive organs, including the liver as well as adipose tissue and skeletal muscle, is involved in the body's energy metabolism [20]. Several studies have demonstrated enhanced SIRT1 content in metabolically relevant hypothalamic nuclei in mice subjected to caloric restriction [46]. SIRT1 expression has been suppressed in both obese rodents and humans $[47,48]$, and active hypothalamic SIRT1 was demonstrated to improve IR in obese rats [49]. In recent studies, the deacetylation activity of SIRT1 has been shown to play a vital role in regulating food intake and energy metabolism [50]. The anorexigenic and anti-obesity function of POMC neurons is required for normal autonomic adaptations of SIRT1 deacetylase [51]. More importantly, FOXO1 is the key deacetylation substrate for SIRT1, which links modulation between downstream appetite-related peptides (NPY and POMC) and SIRT1 [52].

In the present study, we designed 6 groups to investigate the possible mechanisms by which EA improves obesity and IR by suppressing appetite. Standard-diet-induced normal rats were placed into the NG group as controls, while HFDI obese and IR rats were set up as models. We established groups receiving EA plus intracerebroventricular injection of EX-527 to investigate whether the SIRT1 antagonist could specifically attenuate the activity of EA. We established intracerebroventricular administration of the agonist SRT1720 alone as the positive control for EA in order to investigate the differences between the mechanisms underlying these two processes.

EA, characterized by sustained stimulation and by the stability and controllability of its parameters, has been applied extensively in research and clinical practice. Without electrical stimulation, it is hard to perform a standardized stimulus in acupuncture which may also be effective. In our study, weight loss after EA stimulation is likely not stress related, as this was partially reversed by central SIRT1 inhibition. Its effectiveness is frequency dependent: $2 \mathrm{~Hz}$ of EA selectively activated the ventral and bottom thalami, while $100 \mathrm{~Hz}$ activated the dorsal thalamus [53]. Recently, low-frequency EA has been proven to regulate the hypothalamicpituitary-adrenal axis, which was widely applied in the treatment of endocrine diseases [54]. The hypothalamic ARC was more sensitive to low-frequency EA than to high-frequency EA [55]. In our previous study, 2-Hz EA could activate the SIRT1/PGC-1 $\alpha$ pathway to regulate gene expression and enhance mitochondrial activity [28]. Collectively, we selected 2-Hz EA as a frequency for this study. In the theory of traditional Chinese medicine, obesity is associated with a spleen afflicted with sputum dampness, and combined application of ST36, CV4, and CV20 were shown to be effective in alleviating obesity [56]. 
Intracerebroventricular cannulation is an effective way to administer drugs and detect levels of substances throughout the central nervous system (CNS). Compared with direct injection by a microinjector, injection after intracerebroventricular cannulation is characterized by its delayable operation and sustainable intervention, and is therefore widely applied in central administration. The depth of cannulation intended to directly activate the ARC has previously been reported as $8.5 \mathrm{~mm}$ beneath the surface of the skull in rats [57]. In our study, the insertion point of the cannula targeted the dorsal third ventricle of the hypothalamus, which is $3.8 \mathrm{~mm}$ beneath the surface of the skull [34]. The insertion of the cannula to this depth not only ensured that drugs could reach the third ventricle but also prevented damage to the hypothalamic nucleus.

Increasing evidence has suggested that EA is effective in improving obesity and related IR via an anorexigenic function. In accordance with these studies, the present study has demonstrated that 8 weeks of EA treatment suppressed food intake and BW gain in obese and IR rats. Although the PBG increase in obese rats was still within the normal range, it also decreased after EA intervention with no FBG changes. EA also ameliorated obesity-induced hyperinsulinemia, indicating that its effect on regulating PBG may be insulin dependent. The change in IPGTT after intervention was less significant than that in IPITT, suggesting that insulin is more sensitive to EA than glucose. This result may be attributed to intact pancreatic function in obese rats. In addition, the hyperinsulinemic-euglycemic clamp, the authoritative method for assessing insulin sensitivity, indicated that HFDI obese rats had significantly lower glucose consumption in insulin target organs. EA significantly increased whole-body glucose uptake, perhaps through its anorexigenic effect or by activation of the autonomic nervous system [58]. Central administration of the SIRT1 antagonist partially inhibited the effectiveness of EA in suppressing appetite, retarding BW gain, and improving insulin sensitivity, suggesting that activation of SIRT1 in the CNS is one of the mechanisms by which EA exerts its function. The incompleteness with which the SIRT1 agonist blocked the EA effects demonstrated that EA had multiple targets. The specificity of the EA effects on SIRT1 activity is verified by the fact that central administration of the SIRT1 agonist had the same biological effects. By contrast, EA was less effective and sensitive in suppressing appetite and improving IR in obese rats than were agonists, which play a more direct role in the hypothalamus. Overall, specific activation of hypothalamic SIRT1 is one of the underlying mechanisms by which EA improves obesity and regulates metabolic homeostasis.

We performed WB to investigate the mechanism of protein regulation in the hypothalamus. EA could significantly upregulate the low expression of hypothalamic SIRT1 in HFDI rats. Consequently, the high expression of SIRT1-mediated deacetylation activity changed the protein acetylation level of FOX01, which ultimately upregulated protein expression of POMC and downregulated that of NPY. Consistent with our assessments of food intake, obesity level, and insulin sensitivity, the SIRT1 antagonist attenuated EA-induced upregulation of SIRT1 expression, as expected. Meanwhile, rats to which the SIRT1 agonist was centrally administered demonstrated higher SIRT1 expression, in accordance with its high sensitivity and efficiency in improving obesity and IR.

Due to the insufficient protein location and correlation of WB, we conducted doublelabeled IF to investigate the nuclear and neuronal positions regulated by EA. In the hypothalamic ARC, EA upregulated the low SIRT1 expression induced by a high-fat diet, an effect which was also blocked by the SIRT1 antagonist. EA and the SIRT1 agonist had identical effects on the ARC. Consistent with previous studies $[43,44,50]$, the SIRT1 protein expression level regulated the acetylation level of FOXO1, while deacetylated FOXO1 regulated that of the POMC gene, and acetylated FOXO1 regulated that of the NPY gene. Overall, one of the mesoscopic protein locations of EA in regulating food intake and obesity was targeted on the hypothalamic ARC. 
Fig. 5. Mechanisms by which EA affects in the network between the nuclei of the CNS and so regulates food intake. SIRT1, silent mating type information regulation 2 homolog 1; FOX01, forkhead box protein 01; Ac-FOX01, acetylated FOX01; NPY, neuropeptide Y; $\alpha$-MSH, $\alpha$-melanocytestimulating hormone; AgRP, agouti-related protein; MC4R, melanocortin receptor 4; TRH, thyrotropin-releasing hormone; $\mathrm{CRH}$, corticotropin releasing hormone; ORX, orexin; MCH, melanin concentrating hormone; PVN, paraventricular nucleus; LHA, lateral hypothalamic area; ARC, arcuate nucleus.

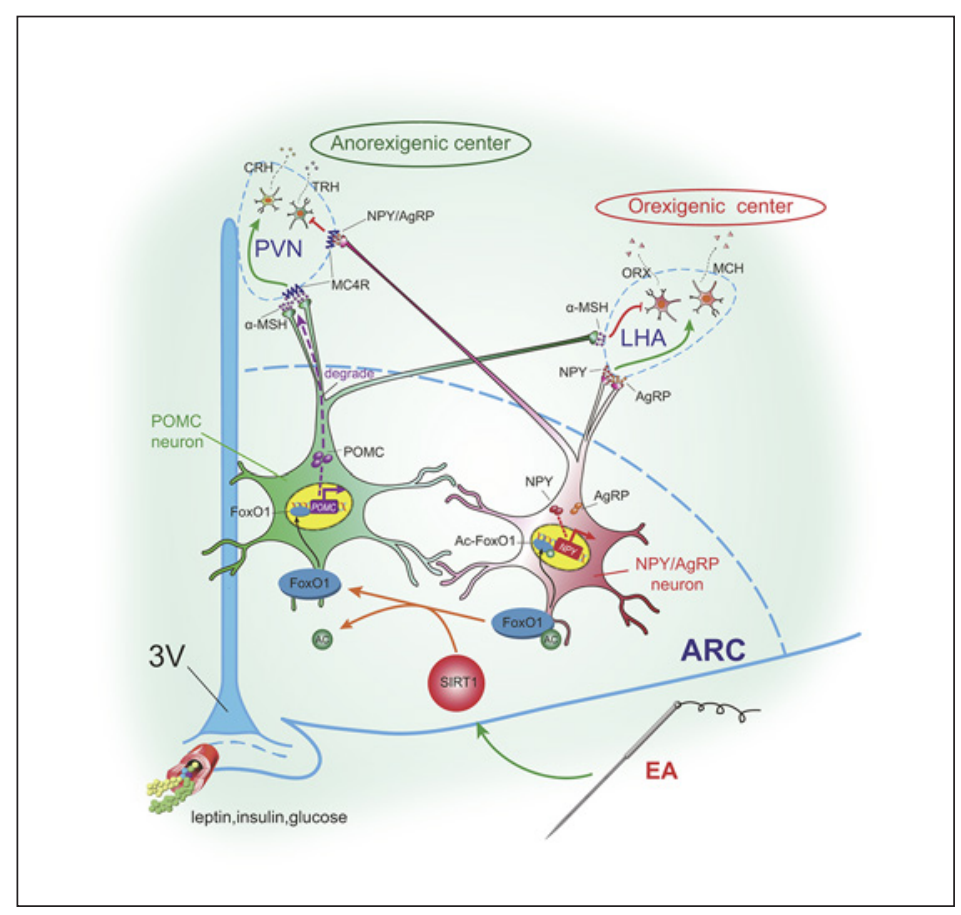

Intriguingly, the results of gene expression in the SIRT1/FOXO1 pathway demonstrated the different mechanisms of EA and the SIRT1 agonist. Considering the different levels of sensitivity in suppressing food intake and improving IR, the SIRT1 agonist could directly upregulate gene expression of SIRT1 and promote protein synthesis in the ARC. By contrast, EA targeted posttranscriptional regulation of the SIRT1 gene, which did not affect gene expression. Neither EA nor the SIRT1 agonist changed the gene expression level of FOXO1, indicating that SIRT1 regulated only the acetylation level of FOXO1 and did not affect its protein synthesis.

Based on previous investigations [28], the present study demonstrated that EA could suppress weight gain and food intake, and improve insulin sensitivity in HFDI obese rats. This study also aimed to determine the potential molecular mechanism in the CNS by which EA causes anorexia and ameliorates IR. EA increased SIRT1 protein expression and upgraded the deacetylation activity in ARC, which downregulated the acetylation level of FOX01. Thus, deacetylated FOXO1 increased gene expression of POMC and suppressed that of NPY, which induced anorexigenic and anti-obesity effects. This mechanism of EA is different from that of the SIRT1 agonist but can achieve similar biological effects. In our previous clinical study, EA was found to be able to activate the vagus nerve [59]. The role of the vagus nerve in transmitting satiety signals from the periphery to the CNS has been well established [60]. Whether EA participates in the vagus nerve-mediated anorectic effect and thus affects the expression of SIRT1 and downstream peptide in the ARC deserves further study.

This study has its share of limitations. Stabilization of Sirt1 protein upon refeeding was induced by decreasing ubiquitination and proteasomal degradation [21]. We did not definitely confirm the mechanism by which EA is involved in this progress. Furthermore, appetite regulation is based on a complex neural network between afferent nerves and the CNS. The neural circuits in the vagus nerve and hypothalamus that may regulate POMC and NPY neurons were found to merit further study. 


\section{Conclusion}

This study suggested a central mechanism by which EA suppresses food intake, improving obesity and IR. Specifically, it promoted protein expression of hypothalamic SIRT1 and mediated the deacetylation level of hypothalamic FOX01, which could upregulate the gene expression of POMC and downregulate that of NPY. This is one important mechanism by which EA exerted an anorexic effect and increases insulin sensitivity. In addition, one of the mesoscopic targets of EA was located in the hypothalamic ARC. Figure 5 illustrates how EA is involved in the network of appetite-related neurons between the nuclei of the CNS and thus regulates food intake. Our study introduced EA as an effective and safe activator of SIRT1, which provided evidence for the application of EA in clinical practice in the treatment of obesity and related IR.

\section{Acknowledgment}

The authors would like to thank the staff members of the Acupuncture and Moxibustion Institute, Hubei University of Chinese Medicine, and the colleagues of the Rehabilitation Medicine Department, Zhongnan Hospital of Wuhan University. Their support helped to successfully complete this study.

\section{Statement of Ethics}

The study was authorized by the Animal Ethics Committee of the Hubei University of Medicine, Wuhan City, Hubei Province, China (online suppl. 1).

\section{Disclosure Statement}

The authors have no conflict of interest to declare.

\section{Funding Sources}

This study received grants from the National Natural Science Foundation of China (Nos. 81473787 and 81001557).

\section{Author Contributions}

F.L. and R.C. conceived the project. Q.S., L.C., and J.L. conceived and designed the experiments. Q.S., L.C. S.W., J.L., and J.L. performed the experiments. Q.S., L.C., and L.X. analyzed the data. Q.S. and F.L. wrote the paper. All authors discussed the results and commented on the manuscript.

\section{References}

1 Organization WH. Obesity and overweight. https://www.who.int/en/news-room/fact-sheets/detail/obesityand-overweight. 2018.

2 Afshin A, Forouzanfar MH, Reitsma MB, Sur P, Estep K, Lee A, et al.; GBD 2015 Obesity Collaborators. Health Effects of Overweight and Obesity in 195 Countries over 25 Years. N Engl J Med. 2017 Jul;377(1):13-27. 
Shu et al.: Acupuncture Targeting SIRT1 and Obesity/Insulin Resistance

3 Collaboration NC; NCD Risk Factor Collaboration (NCD-RisC). Trends in adult body-mass index in 200 countries from 1975 to 2014: a pooled analysis of 1698 population-based measurement studies with $19 \cdot 2$ million participants. Lancet. 2016 Apr;387(10026):1377-96.

4 Williams EP, Mesidor M, Winters K, Dubbert PM, Wyatt SB. Overweight and Obesity: Prevalence, Consequences, and Causes of a Growing Public Health Problem. Curr Obes Rep. 2015 Sep;4(3):363-70.

5 Kahn SE, Hull RL, Utzschneider KM. Mechanisms linking obesity to insulin resistance and type 2 diabetes. Nature. 2006 Dec;444(7121):840-6.

6 Knowler WC, Barrett-Connor E, Fowler SE, Hamman RF, Lachin JM, Walker EA, et al.; Diabetes Prevention Program Research Group. Reduction in the incidence of type 2 diabetes with lifestyle intervention or metformin. N Engl J Med. 2002 Feb;346(6):393-403.

7 Christensen R, Kristensen PK, Bartels EM, Bliddal H, Astrup A. Efficacy and safety of the weight-loss drug rimonabant: a meta-analysis of randomised trials. Lancet. 2007 Nov;370(9600):1706-13.

8 Connolly HM, Crary JL, McGoon MD, Hensrud DD, Edwards BS, Edwards WD, et al. Valvular heart disease associated with fenfluramine-phentermine. N Engl J Med. 1997 Aug;337(9):581-8.

9 James WP, Caterson ID, Coutinho W, Finer N, Van Gaal LF, Maggioni AP, et al.; SCOUT Investigators. Effect of sibutramine on cardiovascular outcomes in overweight and obese subjects. N Engl J Med. 2010 Sep;363(10): 905-17.

10 Bray GA, Siri-Tarino PW. The Role of Macronutrient Content in the Diet for Weight Management. Endocrinol Metab Clin North Am. 2016 Sep;45(3):581-604.

11 Ross R, Hudson R, Stotz PJ, Lam M. Effects of exercise amount and intensity on abdominal obesity and glucose tolerance in obese adults: a randomized trial. Ann Intern Med. 2015 Mar;162(5):325-34.

12 Thomas DM, Bouchard C, Church T, Slentz C, Kraus WE, Redman LM, et al. Why do individuals not lose more weight from an exercise intervention at a defined dose? An energy balance analysis. Obes Rev. 2012 Oct; 13(10):835-47.

13 Kim SY, Shin IS, Park YJ. Effect of acupuncture and intervention types on weight loss: a systematic review and meta-analysis. Obes Rev. 2018 Nov;19(11):1585-96.

14 Cho SH, Lee JS, Thabane L, Lee J. Acupuncture for obesity: a systematic review and meta-analysis. Int J Obes. 2009 Feb;33(2):183-96.

15 Martinez B, Peplow PV: Treatment of insulin resistance by acupuncture: a review of human and animal studies. Acupunct Med. 2016;34:310-319.

16 Tseng CC, Tseng A, Tseng J, Chang CH: Effect of Laser Acupuncture on Anthropometric Measurements and Appetite Sensations in Obese Subjects. Evid Based Complement Alternat Med. 2016;2016:9365326.

17 Schwartz MW, Woods SC, Porte D Jr, Seeley RJ, Baskin DG. Central nervous system control of food intake. Nature. 2000 Apr;404(6778):661-71.

18 Sousa-Ferreira L, de Almeida LP, Cavadas C. Role of hypothalamic neurogenesis in feeding regulation. Trends Endocrinol Metab. 2014 Feb;25(2):80-8.

19 Cohen HY, Miller C, Bitterman KJ, Wall NR, Hekking B, Kessler B, et al. Calorie restriction promotes mammalian cell survival by inducing the SIRT1 deacetylase. Science. 2004 Jul;305(5682):390-2.

20 Liang F, Kume S, Koya D. SIRT1 and insulin resistance. Nat Rev Endocrinol. 2009 Jul;5(7):367-73.

21 Sasaki T, Kim HJ, Kobayashi M, Kitamura YI, Yokota-Hashimoto H, Shiuchi T, et al. Induction of hypothalamic Sirt1 leads to cessation of feeding via agouti-related peptide. Endocrinology. 2010 Jun;151(6):2556-66.

22 Sasaki T, Kikuchi O, Shimpuku M, Susanti VY, Yokota-Hashimoto H, Taguchi R, et al. Hypothalamic SIRT1 prevents age-associated weight gain by improving leptin sensitivity in mice. Diabetologia. 2014 Apr;57(4):819-31.

23 Sin TK, Yung BY, Siu PM: Modulation of SIRT1-Foxo1 signaling axis by resveratrol: implications in skeletal muscle aging and insulin resistance. Cell Physiol Biochem. 2015;35(2):541-52.

24 Kousteni S. Fox01, the transcriptional chief of staff of energy metabolism. Bone. 2012 Feb;50(2):437-43.

25 Kim MS, Pak YK, Jang PG, Namkoong C, Choi YS, Won JC, et al. Role of hypothalamic Foxo1 in the regulation of food intake and energy homeostasis. Nat Neurosci. 2006 Jul;9(7):901-6.

26 Tian N, Wang F, Tian DR, Zou Y, Wang SW, Guan LL, et al. Electroacupuncture suppresses expression of gastric ghrelin and hypothalamic NPY in chronic food restricted rats. Peptides. 2006 Sep;27(9):2313-20.

27 Ji B, Hu J, Ma S. Effects of electroacupuncture Zusanli (ST36) on food intake and expression of POMC and TRPV1 through afferents-medulla pathway in obese prone rats. Peptides. 2013 Feb;40:188-94.

28 Liang F, Chen R, Nakagawa A, Nishizawa M, Tsuda S, Wang H, et al.: Low-Frequency Electroacupuncture Improves Insulin Sensitivity in Obese Diabetic Mice through Activation of SIRT1/PGC-1alpha in Skeletal Muscle. Evid Based Complement Alternat Med. 2011;2011:735297.

29 Kilkenny C, Browne WJ, Cuthill IC, Emerson M, Altman DG. Improving bioscience research reporting: the ARRIVE guidelines for reporting animal research. PLoS Biol. 2010 Jun;8(6):e1000412.

30 Zecchin HG, Priviero FB, Souza CT, Zecchin KG, Prada PO, Carvalheira JB, et al. Defective insulin and acetylcholine induction of endothelial cell-nitric oxide synthase through insulin receptor substrate/Akt signaling pathway in aorta of obese rats. Diabetes. 2007 Apr;56(4):1014-24.

31 Yin CS, Jeong HS, Park HJ, Baik Y, Yoon MH, Choi CB, et al. A proposed transpositional acupoint system in a mouse and rat model. Res Vet Sci. 2008 Apr;84(2):159-65.

32 Zhang J, Huang K, Zhong G, Huang Y, Li S, Qu S, et al.: Acupuncture Decreases NF-кB p65, miR-155, and miR-21 and Increases miR-146a Expression in Chronic Atrophic Gastritis Rats. Evid Based Complement Alternat Med. 2016;2016:9404629. 
Shu et al.: Acupuncture Targeting SIRT1 and Obesity/Insulin Resistance

33 Li M, Zhang Y. Modulation of gene expression in cholesterol-lowering effect of electroacupuncture at Fenglong acupoint (ST40): a cDNA microarray study. Int J Mol Med. 2007 Apr;19(4):617-29.

34 Paxinos G, Watson C. The rat brain. Stereotaxic coordinates. London, UK: Elsevier Inc.; 2007.

35 Chen HZ, Guo S, Li ZZ, Lu Y, Jiang DS, Zhang R, et al. A critical role for interferon regulatory factor 9 in cerebral ischemic stroke. J Neurosci. 2014 Sep;34(36):11897-912.

36 Velásquez DA, Martínez G, Romero A, Vázquez MJ, Boit KD, Dopeso-Reyes IG, et al. The central Sirtuin 1/p53 pathway is essential for the orexigenic action of ghrelin. Diabetes. 2011 Apr;60(4):1177-85.

37 Li X, Yang J, Zhu Y, Liu Y, Shi X, Yang G. Mouse Maternal High-Fat Intake Dynamically Programmed mRNA m(6) A Modifications in Adipose and Skeletal Muscle Tissues in Offspring. Int J Mol Sci. 2016;17(8):E1336.

38 Purkayastha S, Zhang H, Zhang G, Ahmed Z, Wang Y, Cai D. Neural dysregulation of peripheral insulin action and blood pressure by brain endoplasmic reticulum stress. Proc Natl Acad Sci USA. 2011 Feb;108(7):2939-44.

39 Shibata E, Kanno T, Tsuchiya A, Kuribayashi K, Tabata C, Nakano T, et al.: Free fatty acids inhibit protein tyrosine phosphatase 1B and activate Akt. Cell Physiol Biochem. 2013;32(4):871-9.

40 Rubinstein M, Low MJ. Molecular and functional genetics of the proopiomelanocortin gene, food intake regulation and obesity. FEBS Lett. 2017 Sep;591(17):2593-606.

41 Sobrino Crespo C, Perianes Cachero A, Puebla Jiménez L, Barrios V, Arilla Ferreiro E. Peptides and food intake. Front Endocrinol (Lausanne). 2014 Apr;5:58.

42 Pandit R, Beerens S, Adan RA. Role of leptin in energy expenditure: the hypothalamic perspective. Am J Physiol Regul Integr Comp Physiol. 2017 Jun;312(6):R938-47.

43 Kettner NM, Mayo SA, Hua J, Lee C, Moore DD, Fu L. Circadian Dysfunction Induces Leptin Resistance in Mice. Cell Metab. 2015 Sep;22(3):448-59.

44 Hasegawa K, Kawahara T, Fujiwara K, Shimpuku M, Sasaki T, Kitamura T, et al. Necdin controls Foxo1 acetylation in hypothalamic arcuate neurons to modulate the thyroid axis. J Neurosci. 2012 Apr;32(16):5562-72.

45 Susanti VY, Sasaki T, Yokota-Hashimoto H, Matsui S, Lee YS, Kikuchi O, et al. Sirt1 rescues the obesity induced by insulin-resistant constitutively-nuclear Fox01 in POMC neurons of male mice. Obesity (Silver Spring). 2014 Oct;22(10):2115-9.

46 Satoh A, Brace CS, Ben-Josef G, West T, Wozniak DF, Holtzman DM, et al. SIRT1 promotes the central adaptive response to diet restriction through activation of the dorsomedial and lateral nuclei of the hypothalamus. J Neurosci. 2010 Jul;30(30):10220-32.

47 Deng XQ, Chen LL, Li NX. The expression of SIRT1 in nonalcoholic fatty liver disease induced by high-fat diet in rats. Liver Int. 2007 Jun;27(5):708-15.

48 Arab Sadeghabadi Z, Nourbakhsh M, Pasalar P, Emamgholipour S, Golestani A, Larijani B, et al. Reduced gene expression of sirtuins and active AMPK levels in children and adolescents with obesity and insulin resistance. Obes Res Clin Pract. 2018 Mar - Apr;12(2):167-73.

49 Knight CM, Gutierrez-Juarez R, Lam TK, Arrieta-Cruz I, Huang L, Schwartz G, et al. Mediobasal hypothalamic SIRT1 is essential for resveratrol's effects on insulin action in rats. Diabetes. 2011 Nov;60(11):2691-700.

50 Toorie AM, Nillni EA. Minireview: central Sirt1 regulates energy balance via the melanocortin system and alternate pathways. Mol Endocrinol. 2014 Sep;28(9):1423-34.

51 Ramadori G, Fujikawa T, Fukuda M, Anderson J, Morgan DA, Mostoslavsky R, et al. SIRT1 deacetylase in POMC neurons is required for homeostatic defenses against diet-induced obesity. Cell Metab. 2010 Jul;12(1):78-87.

52 Cakir I, Perello M, Lansari O, Messier NJ, Vaslet CA, Nillni EA. Hypothalamic Sirt1 regulates food intake in a rodent model system. PLoS One. 2009 Dec;4(12):e8322.

53 Jin Z, Zhang WT, Luo F, Zhang KL, Zhang L, Zeng YW, et al. [Frequency-specific responses of human brain to peripheral transcutaneous electric nerve stimulation: a functional magnetic resonance imaging study]. Sheng Li Xue Bao. 2001 Aug;53(4):275-80. Chinese.

54 Zhaohui Z, Jingzhu Z, Guipeng D, Xuesong W, Yuanming Z, Yinping W, et al. Role of neuropeptide Y in regulating hypothalamus-pituitary-gonad axis in the rats treated with electro-acupuncture. Neuropeptides. 2012 Jun; 46(3):133-9.

55 Wang K, Zhang R, He F, Lin LB, Xiang XH, Ping XJ, et al. Electroacupuncture frequency-related transcriptional response in rat arcuate nucleus revealed region-distinctive changes in response to low- and high-frequency electroacupuncture. J Neurosci Res. 2012 Jul;90(7):1464-73.

56 Wang JJ, Chen X, Mei-Ling YU, Sheng-Feng LU. Characteristics of meridians and acupoints selection for treatment of simple obesity: a data mining research on latest 10 years. J Beijing Univ Trad Chin Med. 2014;15: 1043-51.

57 Roy C, Roy MC, Gauvreau D, Poulin AM, Tom FQ, Timofeeva E, et al. Acute injection of ASP in the third ventricle inhibits food intake and locomotor activity in rats. Am J Physiol Endocrinol Metab. 2011 Jul;301(1):E232-41.

58 Benrick A, Kokosar M, Hu M, Larsson M, Maliqueo M, Marcondes RR, et al. Autonomic nervous system activation mediates the increase in whole-body glucose uptake in response to electroacupuncture. FASEB J. 2017 Aug;31(8):3288-97.

59 Shu Q, Wang H, Litscher D, Wu S, Chen L, Gaischek I, et al. Acupuncture and Moxibustion have Different Effects on Fatigue by Regulating the Autonomic Nervous System: A Pilot Controlled Clinical Trial. Sci Rep. 2016 Nov; 6(1):37846.

60 de Lartigue G. Role of the vagus nerve in the development and treatment of diet-induced obesity. J Physiol. 2016 Oct;594(20):5791-815. 